\title{
LA NOVELLISATION EN VERSION ILLUSTRÉE
}

\author{
Avec ou sans images
}

\author{
Jan Baetens ${ }^{1}$
}

\section{Un genre, deux univers}

On appelle novellisation la transposition en livre (de fiction) d'un scénario ou d'un film original. Ces dernières années, plusieurs études ont rendu plus visible un genre certes très lu, mais totalement ignoré par les approches traditionnelles de la littérature, hostiles ou méfiantes envers ce qui n'appartient pas au canon du jour. ${ }^{2}$ La prise de conscience, notamment sous l'effet des études culturelles, du caractère instable et relativement subjectif de ce qu'on entend par « littérature », " canon », " patrimoine », etc., et la valorisation progressive des divers types de paralittérature - de la bande dessinée au roman pornographique, du polar aux livres pour adolescents- ont jusqu'ici eu peu d'impact sur la reconnaissance d'un genre dont on sait pourtant qu'il est aussi ancien

1 Jan Baetens est professeur à la Katholieke Universiteit Leuven (KUL) à l'Instituut voor Culturele Studies.

2 Voir surtout Jan Baetens et Marc Lits, dir., La Novellisation. Du film au livre. Novelization. From Film to Novel, Leuven, Louvain University Press, 2004, et Jan Baetens, La novellisation, Du film au roman. Bruxelles, Les Impressions Nouvelles, 2008 .

Recherches en communication, $\mathrm{n}^{\circ} 31$ (2009). 
que le cinéma et aussi divers que la production cinématographique ellemême.

Que signifie une telle carence, apparemment peu compatible avec nos intuitions sur le public contemporain « omnivore » $?^{1}$ Le refoulement persistant de la novellisation comme objet d'étude " sérieux », du moins en comparaison avec d'autres sujets longtemps jugés marginaux ou méprisables, révèle immédiatement les limites de notre approche libérale et postmoderne du fait culturel. Qu'à l'époque du « anything goes » certaines pratiques continuent à souffrir d'une mise à l'écart radicale, devrait nous inciter à plus de prudence quant aux idées sur les rapports entre culture savante et culture médiatique -si l'on nous permet de traduire ainsi l'antinomie du « haut » et du «bas » ou encore du « légitime » et du " populaire ». De son côté, le manque d'intérêt pour un phénomène social et commercial aussi important que la novellisation, qui est tout sauf un créneau mineur dans le monde de l'édition, signale aussi les limites de notre tolérance d'omnivores à l'égard de certaines formes culturelles. Mais précisions l'un et l'autre de ces points.

En ce qui concerne tout d'abord la question du « haut » et du « bas », l'exemple de la novellisation montre bien que les critères de jugement, de goût et de distinction ne discriminent plus les genres ou les médias (littérature versus cinéma, musique classique versus polar, etc.) puisque tous bénéficient aujourd'hui de la même attention (du moins en théorie). On observe par contre que ces mêmes critères se reproduisent à l'intérieur de chaque domaine, qu'ils stratifient de manière aussi draconienne que dans le système traditionnel où cette stratification portait sur les médias et les genres. On est donc loin de la situation tant redoutée par Alain Finkielkraut et consorts, qui s'émouvaient de voir mélangés Shakespeare et Tintin. Si l'auteur de La Défaite de la pensée se trompe sur ce point, ce n'est pas parce que le public apprécie davantage l'un (Tintin) que l'autre (Shakespeare), mais parce que, à l'intérieur de ces deux corpus, tout ne se vaut pas. Certaines pièces de Shakespeare ou certains albums de Tintin sont jugés meilleurs que d'autres, et cette différenciation interne est exactement la même que celle qui, à une époque antérieure, plaçait le théâtre au-dessus de la

1 Pour plus de détails, voir Jan Baetens, « La culture populaire n'existe pas, ou les ambiguïtés des Cultural Studies », in Hermès, No 42, 2005, pp. 70-77. 
bande dessinée. La novellisation illustre ces deux types de distinction: le genre est dévalué en bloc, mais en même temps il sera aussi le théâtre d'une série de différenciations internes très précises.

En ce qui concerne ensuite la question du public omnivore, la novellisation rappelle clairement que l'élasticité du goût postmoderne n'est pas sans limites. On s'en était rendu compte dès les premières enquêtes sur les préférences musicales ${ }^{1}$ : d'une part, la diversité des goûts et l'hybridité des choix s'arrêtent face aux extrêmes que sont, du côté de la culture traditionnelle, le ballet, exemple même du kitsch irrécupérable, et, du côté de la culture populaire, certaines formes de «métal hurlant », expression musicale elle aussi difficilement intégrable ; d'autre part, l'attitude « ouverte » de l'omnivore est loin d'être partagée par toutes les couches du public, puisqu'elle semble caractériser avant tout la consommation culturelle du public mieux éduqué. Dans le domaine du cinéma, pour prendre aussi un exemple non musical, le clivage entre cinéma d'art et essai et cinéma commercial tend même à se renforcer, y compris au niveau des modes de consommation ou de visionnement, avec par exemple un taux de fréquentation des salles anormalement bas pour les spectateurs à capital scolaire réduit (pourtant grand amateur de cinéma, mais à la télévision seulement) et une désaffection croissante du public cultivé à l'égard des multiplexes et, dans une moindre mesure, des salles art et essai (boudées au profit du DVD).

Les préjugés à l'égard de certaines formes culturelles sont donc plus tenaces que ne laissent croire les idées reçues sur le grand brassage postmoderne. La littérature ne fait pas exception à cette règle, témoin la cécité culturelle des chercheurs face à des secteurs aussi vivants que la littérature régionale, le roman historique populaire ou la novellisation, entre autres. S'agissant de cette dernière, l'écart est net entre sa présence indéniable sur les tables des libraires, dans les points de vente alternatifs ou encore dans les campagnes de publicité et de merchandising des nouveaux films, d'une part, et la reconduction des réactions très méprisantes dont il fait toujours l'objet, d'autre part. Autant les

1 Voir par exemple les analyses des résultats pour la Flandre : John Lievens \& Hans Waege (dir.), Cultuurkijker - Cultuurparticipatie in breedbeeld. Eerste analyses van de survey cultuurparticipatie in Vlaanderen 2003-2004, Bruxelles, De Boeck, 2005 (partiellement accessible via Google Books). 
novellisations se multiplient et se vendent, parfois très bien du reste, ${ }^{1}$ autant les réactions du monde littéraire gardent un caractère prévisible et stéréotypé, entre dénigrement et réappropriation. On se moque de la mauvaise qualité de ces ouvrages et lorsque de " vrais » auteurs en écrivent, c'est toujours sur commande, jamais par goût, et l'anonymat ou le pseudonyme sont de rigueur En même temps, on essaie d'inventer des formes « littéraires » du genre, qui s'opposent le plus possible aux formules connues. Etablir l'inventaire de ces différences n'est pas difficile. Tout d'abord, une novellisation à vocation littéraire se distingue de son équivalent populaire par le choix du film transposé : la novellisation populaire, produit jetable qui sort en même temps que le film et termine sa carrière dès que celui-ci n'est plus à l'affiche, porte par définition sur le cinéma commercial d'aujourd'hui, dont le statut culturel est encore en suspens ; la novellisation littéraire se donne des objets de choix, à savoir des œuvres à réputation cinéphilique, que les auteurs ont vues dans leur adolescence-c'est-à-dire à l'âge où l'on est le plus marqué par le cinéma- et qu'ils ont eu tout temps de transformer en objets mythiques (par exemple La Nuit du chasseur de Charles Laughton, Le limier de Joseph Mankiewicz ou Chaînes conjugales du même²). Ensuite, une novellisation populaire veut d'abord raconter un récit, sans fioritures ni temps faibles (qu'on appelle parfois « descriptions »), tandis qu'une novellisation littéraire se plaît souvent à ralentir le rythme et, plus encore, à thématiser la situation narrative, laquelle n'est jamais transparente : au lieu de raconter l'histoire du film à la troisième personne avec énormément de dialogues (n'oublions pas que les novellisations populaires ne sont parfois que des scénarios à peine " habillés »), on introduit un narrateur décrivant ses propres pensées et réactions au cours d'une projection ou d'un visionnement privé de l'œuvre. Enfin, contrairement à la novellisation populaire en prose, les novellisations littéraires se laissent séduire par le prestige de l'écriture poétique, s'offrant ainsi la possibilité de jouer sur un régime de lecture qui, comme le disait Mallarmé, " détourne l'oisif ». Voici, à titre d'exemple, les premières lignes d'un poème -du reste fort réussi- de Philippe Beck,

1 Un des bestsellers flamands de 2008 était le Journal de Sara, héroïne d'une " télénouvelle » américaine adaptée aux goûts du public-cible de la chaîne privée VTM. A l'échelle mondiale, on peut mentionner le succès de Spiderman III.

2 Respectivement : Pierre Alféri, Le cinéma des familles (Paris, P.O.L, 1999), Tanguy Viel, Cinéma (Paris, Minuit, 1999) et Alice Ferney, Paradis conjugal (Paris, Albin Michel, 2008). 
où beaucoup doit être deviné par le lecteur, à commencer par le titre du film (Voyage en Italie, 1953) et les aspects de l'œuvre sélectionnés par un texte, pour citer encore Mallarmé, «le récit s'évite » :

99

\section{A Roberto Rossellini}

L'île d'Ischia, résumé de bains de soufre, apparaît de la terrasse des durs qui voyagent en Italie au prix du Musée.

Ebullition et ionisation : amplification de la fumée par la torche. Fumée simultanée dans la pente autour de la torche.

Cicérone charrie la légende des yeux et des muscles durs grâce à la politique, l'art de ne rien regretter. $(. . .)^{1}$

\section{Texte-image, allers-retours}

Toutefois, la caractéristique la plus fondamentale des novellisations littéraires est leur rapport très singulier à l'illustration. Quelle que soit son orientation, littéraire ou populaire, la novellisation n'est pas un genre qui cherche à faire concurrence au pouvoir visuel du cinéma. Elle se caractérise par un déficit visuel certain. Le novellisateur décrit peu, même si les raisons ne sont pas toujours les mêmes. Dans la novellisation populaire, souvent écrite par des auteurs qui ont pas vu le film encore en gestation, on craint que les descriptions n'agacent ou ne déroutent le lecteur. Dans la novellisation littéraire, l'accent est mis sur les traits spécifiques de la parole littéraire, ce qui exclut toute tentative de copier servilement les images du film. Cependant, cette anti-visualité est loin d'être absolue, car il arrive que les novellisations soient illustrées, à la fois à l'intérieur du livre, en des pages hors-texte ou en des cahiers d'illustrations, et en couverture, recto et verso confondus. Telle est en tout cas la norme dans les novellisations populaires et commerciales, qui sur ce point aussi se séparent de la novellisation littéraire, elle souvent rétive au principe de l'illustration.

1 Élégies Hé, Courbevoie, Théâtre typographique, 2005, p. 124. 
La forme et le fonctionnement des illustrations en régime de novellisation populaire expliquent facilement les réticences des novellisations littéraires. Trois éléments y reviennent systématiquement. Le premier touche à la fonction des images, qui est commerciale. Leur but est moins de reproduire le déroulement du film que d'établir un contact très fort avec le film dans son ensemble, que le lecteur est censé connaître déjà -ou dont il est invité à prendre connaissance sans tarder (le message premier d'une novellisation n'est donc pas « achetez-moi », mais au contraire « est-ce que vous m'avez déjà vu ? »). Le second trait est en rapport avec le type d'image retenu : de plus en plus, l'illustration se rapproche de l'affiche du film, ce qui court-circuite encore davantage le lancement du film et la mise en vente du livre. Le troisième aspect, qui combine et creuse les deux premiers, concerne le brouillage entre texte et péritexte. En effet, à l'intérieur des livres, s'il leur arrive d'être illustrés (ce qui est loin d'être toujours le cas), les photos n'ont souvent qu'une fonction narrative réduite, car au lieu de visualiser les épisodes du récit, elles mettent en avant les personnages, ou plus exactement les comédiens. Arrachant les photos au texte pour les rapprocher de la couverture, ce glissement n'est pas étranger aux contraintes de forme et de fonction qui pèsent sur la novellisation commerciale illustrée. Ce qui doit s'illustrer, ce n'est pas le récit du film, mais le film lui-même -et dans le cinéma populaire, un film n'est pas séparable des comédiens qui le portent. Par ce rappel du film l'illustration obéit logiquement, le cinéma étant aussi une industrie, à un but publicitaire. Enfin, la meilleure façon d'établir le lien entre le livre et le film est de passer par les images qui résument le mieux l'œuvre cinématographique, soit l'affiche du film et les portraits des stars, car même si on n'a pas vu le film, il est probable qu'on connaît et l'affiche et les comédiens.

Il ne faut donc pas s'étonner que les illustrations des novellisations populaires deviennent comme naturellement autre chose que le double visuel du récit proposé par le texte. Beaucoup de ces images ne sont pas des photogrammes du film même, mais des photos de tournage, que l'on aurait du mal à retrouver, même approximativement, dans l'œuvre projetée. Qui plus est, l'esthétique de ces photographes peut être différente de celle des films qu'elles sont supposées illustrer. Dans le cas du cinéma français des années 40 et 50, par exemple, il est clair que les photos des vedettes de l'époque tendent à respecter très fortement l'idéal façonné par les studios Harcourt, brillamment décrit par Roland 
Barthes dans une de ses mythologie ${ }^{1}$ et dont il est passionnant d'étudier les prolongements à l'ère digitale. ${ }^{2}$ Format, costume, cadrage, éclairage, pose, tous ces éléments font voir que les illustrations ne reproduisent pas prioritairement l'œuvre filmique. Il s'y ajoute que l'ordre dans lequel sont présentées ces images ne correspond pas toujours à la diégèse du film. Même si la novellisation commerciale, inévitablement soucieuse des habitudes de lecture conventionnelles et de structures narratives ayant un début, un milieu et une fin, recule devant des ruptures trop voyantes, il existe des principes généraux qui n'ont rien de narratif au sens strict du terme. Ainsi on commencera toujours par insérer les photos des vedettes, d'abord seules, puis en groupe, puis en couple (car tout film classique est un film d'amour). Plus que l'ordre de la diégèse,

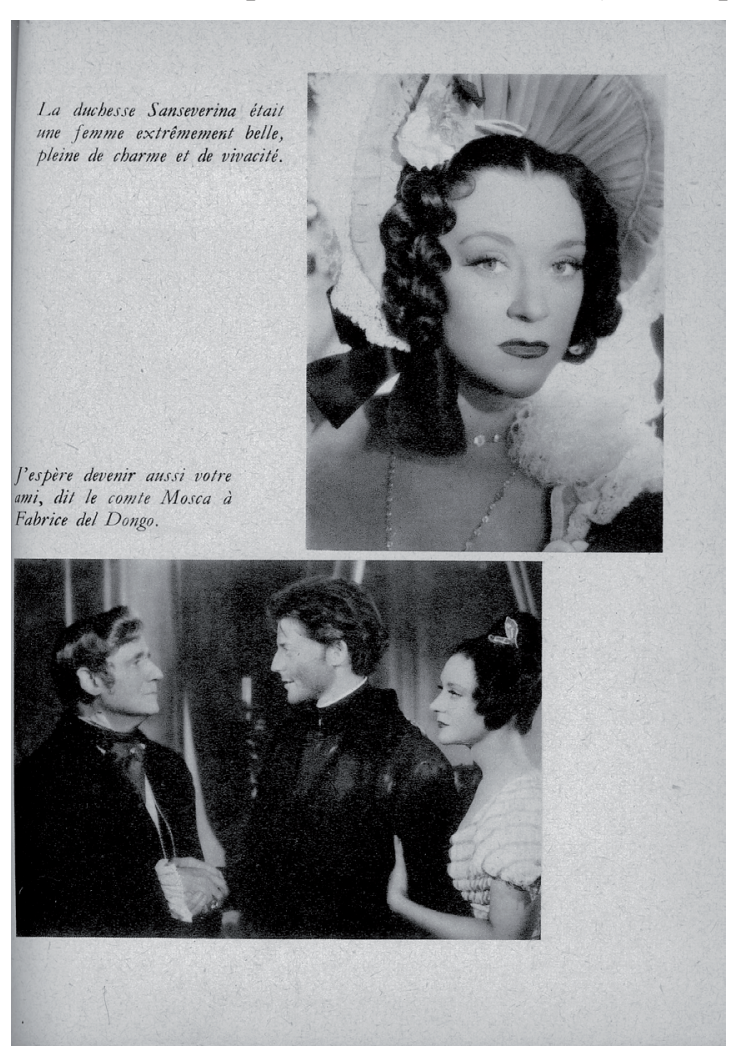
ce genre d'enchaînements respecte les contraintes du pacte romanesque à sceller avec le lecteur.

A titre d'exemple, voici les trois premières images d'une novellisation représentative: la récriture par Christian Véry de La Chartreuse de Parme, adapté à l'écran par Christian Jacque, avec Gérard Philippe dans le rôle de Fabrice del Dongo, Maria Casarès dans le rôle de la duchesse Sanseverina, et Renée Faure dans le rôle de Clélia Conte ${ }^{3}$ :

1 «L'acteur d'Harcourt », in Mythologies, Paris, Seuil, 1957 (cité d'après l'édition Points), pp. 24-27.

2 Voir Bernard Darras, « Sémiotique pragmatique et photographie numérique. Le cas de la retouche photographique » (à paraître dans $R S / S I$ ).

3 Paris, éditions SELF, 1948. 


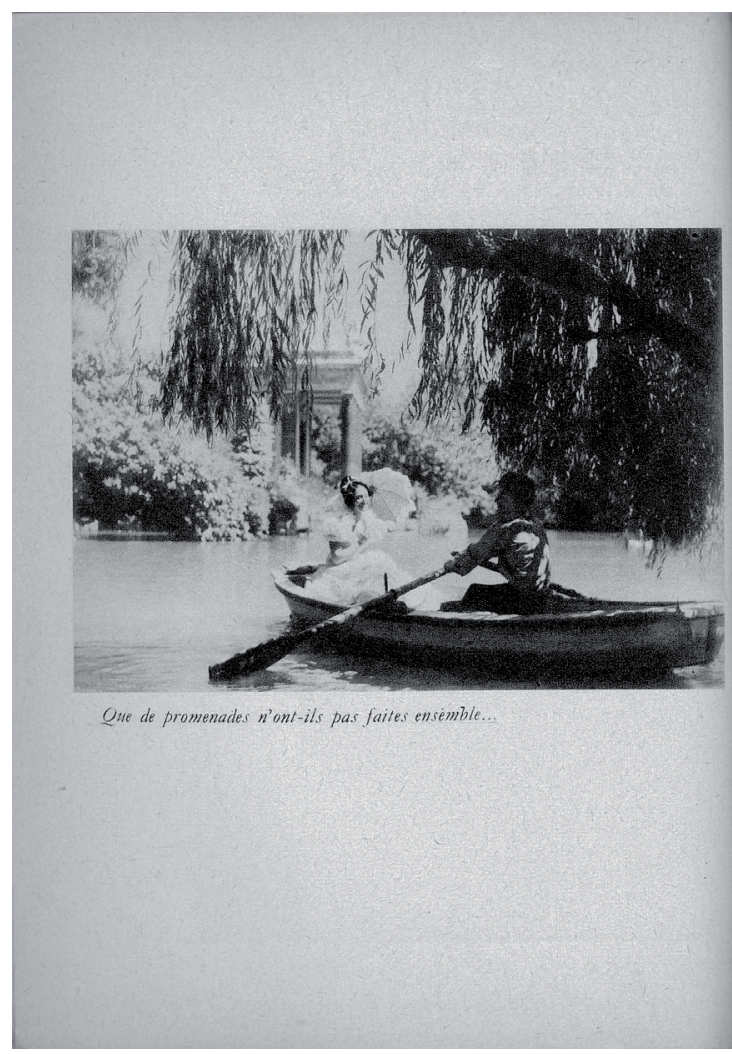

On y reconnaît sans peine les trois phases distinguées ci-dessus : la vedette, le groupe, la rencontre.

Il ne faut pas s'étonner non plus que les illustrations des textes à ambition plus littéraire s'opposent radicalement à ces conventions. Trois formules, la première fréquemment attestée, les deux autres plutôt rares, peuvent ici être soulignées. D'abord, l'absence d'illustrations, en couverture comme à l'intérieur -solution radicale mais efficace pour marquer l'écart entre classes et catégories de novellisation. Ensuite le changement de média : le passage de la photographie au dessin, celui-ci n'ayant pas les mêmes connotations que celle-là, puisque un dessin passe pour plus artistique qu'une photographie, du moins dans la perception traditionnelle des types d'images. C'est par exemple la solution retenue pour les deux novellisations de Jacques Tati par Jean-Claude Carrière, qu'illustrent les dessins de Pierre Etaix, concepteur de l'affiche du film ${ }^{1}$ Enfin, l'usage de vrais photogrammes, en lieu et place des photos de tournage ou des portraits de studio. C'est le système utilisé par Chris Marker dans ses expériences photoromanesques, de La Jetée remise en pages par Bruce $\mathrm{Mau}^{2}$ au récent Staring Back, qui oscille entre plusieurs médias ${ }^{3}$. C'est

1 Les Vacances de M. Hulot, Paris, Laffont, 1958, et Mon Oncle, Paris, Laffont, 1959.

2 New York, Zone Books, 1992 (édition bilingue).

3 Staring Back, Cambridge, Mass et Columbus, Ohio, MIT Press et Wexner Center for 

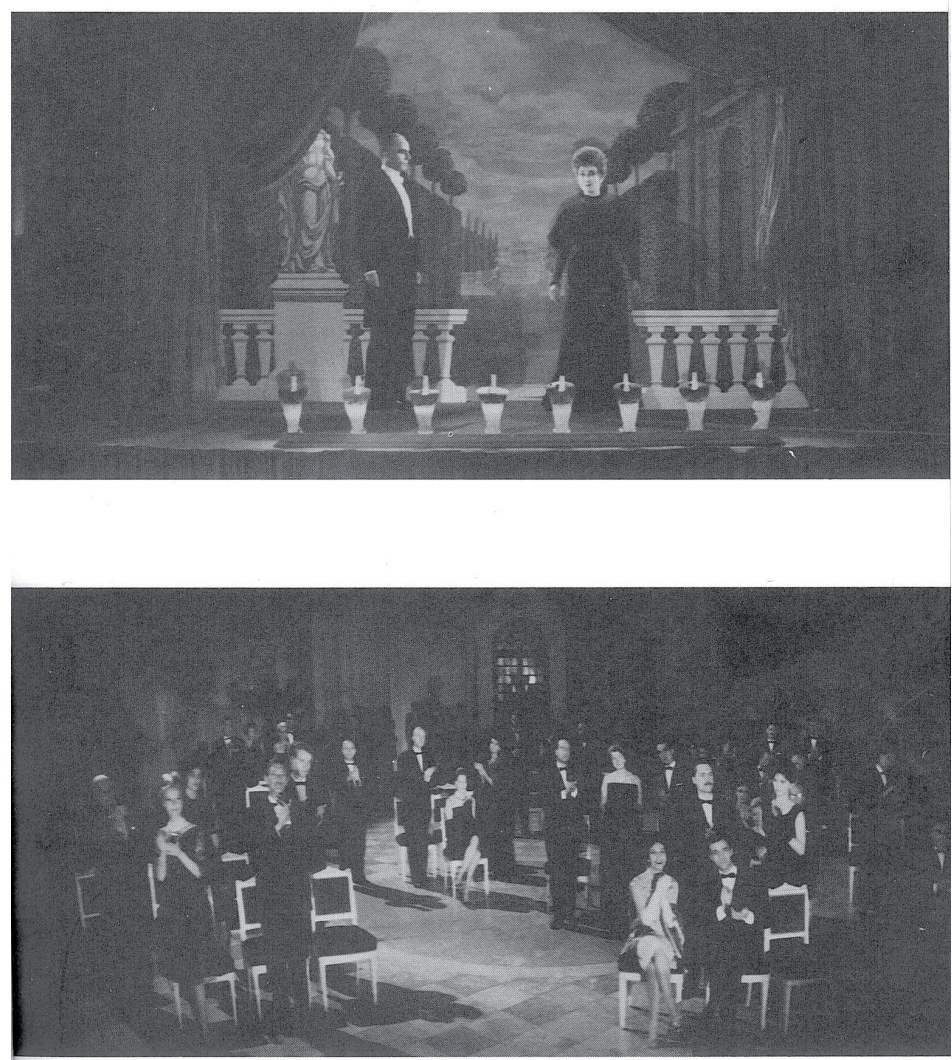

La salle du théâtre à la fin de la représentation. (P. 32.)

aussi, de façon plus discrète mais non moins efficace, la voie choisie par Alain Robbe-Grillet, notamment dans ses premiers ciné-romans L'Année dernière à Marienbad ou L'Immortelle ${ }^{1}$. Dans ces deux livres, les images s'éloignent formellement des photos de plateau (le format et surtout le cadrage sont ceux d'une image cinématographique, avec relativement peu de plans rapprochés et bon nombre de vues panoramiques). Elles obtempèrent aussi à une tout autre logique. Au début de L'année dernière à Marienbad, par exemple, l'important n'est plus d'introduire d'abord les vedettes, puis les vedettes, puis les héros fictifs qu'elles incarnent, enfin les rapports amoureux qui vont se nouer entre

the Arts, 2007.

1 Publiés respectivement en 1961 et 1963, aux éditions de Minuit (Paris). 
les personnages au sein de la diégèse, mais de confronter le lecteur avec un univers de représentation (on est au théâtre, vu de la salle, puis de la scène), puis d'attirer son attention sur les principes formels du montage des photos sur la page (en l'occurrence la technique cinématographique du champ-contrechamp). ${ }^{1}$

\section{Le cinéma et la littérature revus par la novellisation}

Ces premières lectures du genre de la novellisation aboutissent à des conclusions périlleuses : systématiquement, en effet, on a mis en exergue l'abîme qui sépare les deux classes de novellisations. Menée dans cette perspective, l'étude de la novellisation risque d'être contreproductive, puisque au nom d'une attention nouvelle aux pratiques littéraires non canoniques, on en revient à renforcer l'opposition qu'on cherchait à dépasser. Or, l'étude des parties illustratives de la novellisation peut conduire vers d'autres analyses, plus voisines de l'esprit des études culturelles. Pour ne pas alourdir l'analyse, on se limitera ici à trois domaines : les théories de l'adaptation ; l'histoire du cinéma ; l'émergence d'une littérature proprement " graphique ».

Tout d'abord, l'étude des novellisations illustrées pose à nouveau la question des rapports entre texte et image ${ }^{2}$. Il est généralement admis que le passage du livre au film heurte la sensibilité du lecteur moyen que le mouvement inverse. La littérature, dit-on, peut certes décrire des lieux, des actions, des objets et des personnages, mais ses descriptions laissent toujours une grande marge de manœuvre à l'imagination du lecteur, qui peut « compléter » à sa guise le portrait d'un héros ou d'une héroïne, par exemple. Si « Madame Bovary, c'est moi », il n'y a pas deux lecteurs qui se font la même idée, ou plus exactement la même

1 A cet égard il est important de signaler que l'édition de poche du même texte inverse complètement le dispositif de l'œuvre originale : au lieu d'avoir une couverture blanche comme l'édition originale, l'édition de poche présente en couverture un dessin de couleur ( le film même, rappelons-le, est en noir et blanc), et tandis que l'édition originale contient de nombreuses illustrations en hors-texte, l'édition de poche n'en a aucune. On peut imaginer que ces différences tiennent à des questions de copyright, mais comment ne pas être sensible à l'aspect parodique de cette adoption du code « savant » pour le dérivé « populaire » de l'œuvre ?

2 Pour une mise au point régulière des questions en ce domaine, voir la nouvelle revue Adaptation. The Journal of Literature on Screen Studies (Oxford University Press, depuis 2008). 
représentation visuelle, du personnage de Flaubert -sauf peut-être s'ils ont vu le film tiré du livre, lu une version photoromanesque, ou assisté à une représentation théâtrale de l'œuvre... On allègue généralement le conflit entre la liberté offerte au lecteur et les contraintes imposées au spectateur pour expliquer l'opposition de certains écrivains à toute forme d'illustration ou d'adaptation et la déception, soi-disant inévitable, qui attend les amateurs de littérature quand ils découvrent les transpositions visuelles de leurs textes préférés. La novellisation, et surtout la novellisation illustrée, bouleversent ces lieux communs. Contrairement à d'autres types de roman, où le recours aux illustrations photographiques demeure exceptionnel, ${ }^{1}$ la novellisation n'a pas peur de juxtaposer « monstration » et « description $» .^{2}$ On pourrait objecter ici que ce mélange résulte du statut secondaire du texte, postérieur et non pas antérieur à l'image, mais cette réserve est un peu courte, dans la mesure où nos idées sur la lecture d'un texte littéraire s'appliquent aussi à nos rapports imaginaires avec le cinéma. De la même façon que les mots sur la page s'ouvrent à des interprétations très diverses, l'image projetée ne coïncide jamais avec telle quelle avec l'image reçue, qui est assimilée et transformée par le spectateur selon des mécanismes idiosyncratiques. Le personnage « universel » de Madame Bovary a beau prendre la forme « particulière » d'Isabelle Huppert dans le film de Claude Chabrol (1991), cette particularisation ne joue qu'au niveau de l'image-objet, non à celui de l'image perçue lors d'une expérience spectatoriale vivante qu'enrichissent ou entravent, nuancent et compliquent des facteurs intellectuels, cognitifs et émotionnels très divers. En ce sens, le rappel de l'image-objet par voie d'illustration devrait gêner davantage que son éloignement, apte, lui, à stimuler l'investissement psychologique du lecteur. Que la novellisation, malgré tout, ne proscrive pas les images, révèle que d'autres mécanismes entrent en jeu. Dans sa forme classique, l'adaptation suppose un avant et un après, un modèle et un résultat, une « source » et une « cible ». Dans une telle optique, le sens de l'orientation est capital : adapter un texte à l'écran n'est pas la même chose que novelliser un film, ni du point de vue technique, ni du point de vue imaginaire. Par contre, dans sa forme

1 Cf. Jan Baetens et Hilde Van Gelder, « Petite poétique de la photographie mise en roman (1970-1990) », in Danièle Méaux (dir.), Photographie et romanesque, Caen, Lettres Modernes Minard, 257-271.

2 Pour utiliser l'utile terminologie d'André Gaudreault dans son ouvrage Du littéraire au filmique, Paris, Armand Colin, 1999. 
nouvelle, l'adaptation se généralise et perd en quelque sorte ses repères temporels. De temporelle, l'adaptation devient spatiale, sa logique se métonymise : les différentes formes sont coprésentes, et ce qui compte n'est plus l'ordre dans lequel s'effectuent les opérations, mais la capacité d'une forme médiatique de se connecter à d'autres formes. Dans ce régime, le critère distinctif n'est plus la distinction entre un avant et un après, mais entre l'isolat et le réseau. Virtuellement, toute forme peut ressurgir ailleurs, sous d'autres apparences médiatiques, fidèles ou non, représentatives ou non, répétitives ou non, mais certaines formes y parviennent plus facilement que d'autres. C'est là une première leçon de l'analyse des novellisations.

Un second axe de recherches concerne la définition du cinéma. A première vue, la novellisation ne semble pas apporter grand-chose à une nouvelle conceptualisation du septième art, mais cette impression est trompeuse. Comme elle met en avant la dimension narrative du texte, la novellisation renforce l'idée conventionnelle que le cinéma est lui aussi un média essentiellement narratif -chose qui a pu paraître l'évidence même à un moment donné de l'histoire du cinéma, mais que l'exhumation du cinéma des premiers temps (qui était un cinéma d'attractions, comme l'ont baptisé Tom Gunning et André Gaudreault), puis l'avènement du post-cinéma (qui n'est pas le cinéma numérique en lui-même mais le dépassement de la doxa narrative tel que l'ont rendu possible les expériences du numérique) ont sérieusement ébréchée. Or, l'insistance sur le récit corrobore non seulement l'idée du cinéma comme média narratif, elle rapproche aussi le cinéma de la littérature. En effet, puisque le récit est de l'ordre du paraphrasable, tout récit cinématographique apparait comme le double d'un récit textuel et bascule donc du côté de la littérature -et peu importe ici que cette intégration du filmique au littéraire se pense de manière réactionnaire ou moderne, qu'on réduise le cinéma à une sorte de littérature en images (version réac) ou qu'on le présente au contraire comme le média appelé à prendre le relais d'une forme d'expression, la littérature, à bout de souffle (version « camérastylo »). La seule comparaison des deux types de récit, littéraire et cinématographique, accroît le rôle dominant du narratif à l'intérieur du cinéma. En ce sens, la novellisation est un genre conservateur, en dépit des formes souvent très novatrices qu'il lui arrive de défricher. Toutefois, l'ajout d'illustrations modifie le statut du genre, même dans ses exemples les plus convenus. En effet, les photos d'accompagnement obligent à penser le cinéma en termes d'images, et non plus en termes de récit. 
Dénarrativisées de manière parfois assez violente, étalant des personnages hors tout contexte, juxtaposées plutôt que mises en séquence, les images de la novellisation débouchent sur un écrasement du potentiel narratif des photos de tournage ou des portraits de studio. Une telle observation n'est nullement banale. Si un film, c'est-à-dire un objet supposé narratif, s'assimile à un matériau visuel où l'aspect narratif coupé de toute base narrative, on peut se demander quel est vraiment le film qu'on voit en salle ou ailleurs, surtout, quel est le film dont on se souvient ? S'il est vrai qu'on se rappelle mieux certaines images du film que l'histoire du film même, faut-il envisager ces images comme une sorte d'aide-mémoire, comme tremplin d'un imaginaire narratif mis en branle par le retour de quelques images-phare, ou convient-il en revanche de souligner la métamorphose d'une suite d'images mobiles en une collection d'images fixes, arrachées au flux temporel et narratif? La question se fait plus prégnante encore quand on passe des images du film projeté aux images du cinéma qui envahissent notre regard et notre mémoire sans visionnement préalable. Or telle est bien aujourd'hui la manière dont nous consommons le cinéma, et on retrouve ici le rôle premier et non plus secondaire ou dérivé de l'adaptation. Comme l'écrit Victor Burgin :

Phénoménologiquement partant, le champ visuel du quotidien dans les cultures "occidentales" contemporaines (mais il en va de même au Japon) est hétérogène et hybride. Le consommateur d'images tourne sans fin les pages des magazines, il surfe sur les vagues des spectacles télévisuels. Il est rare que l'intégrité de l'objet sémantique soit respectée. De plus, les frontières de l' « objet » même sont élargies, elles deviennent perméables, se transforment de mille et une manières. Un film, par exemple, est un objet dont on fait la connaissance à travers des posters, des slogans et autres formes de publicité, comme les bandes-annonce ou les clips TV; on apprend à le connaître par des comptes rendus de journaux, des abstracts dans des ouvrages de références ou peut-être des articles théoriques (avec leur leurs assemblages de séquences de photogrammes); par des photographies de production, des agrandissements d'images, des gadgets et ainsi de suite. En mettant bout à bout ces fragments métonymiques dans notre mémoire, nous pouvons finir 
par avoir l'impression de bien connaître un film qu'en fait nous n'avons jamais $\mathrm{vu}^{1}$.

Tel est le régime notre perception du cinéma aujourd'hui-et l'avantage des novellisations illustrées, pour banales qu'elles puissent être, est de proclamer tout haut l'hybridité médiatique de notre regard sur le film. On doit dès lors prendre plus au sérieux les mille et une variations textuelles et visuelles sur les objets cinématographiques qui circulent dans la culture médiatique contemporaine. Comme l'ont bien analysé Edouard Arnoldy et Laurent Le Forestier dans leur étude des novellisations illustrées de Tallandier, notamment Tire-au-flanc de Jean Renoir (1928), l'escorte iconographique s'est alliée au texte pour neutraliser, voire casser ce que le travail du metteur en scène a pu produire de plus inventif, de moins facile. Ayant posé que " photographies et récit constituent deux séries autonomes qui, telles des sinusoïdes, se recoupent parfois mais sans jamais se confondre $»^{2}$, ils déduisent très justement des différences que l'intervention des illustrateurs est tout sauf innocente : en « corrigeant » certaines caractéristiques du film de Renoir qu'ils considèrent comme une " erreur », ces illustrateurs transforment non seulement notre perception de Tire-au-flanc, mais en reviennent « à l'ère industrielle du cinéma ", à 'une époque d'avant l'auteur', c'est-àdire avant la reconnaissance culturelle du cinéaste comme auteur $»{ }^{3}$

Toutes proportions gardées, mais de façon inverse, des tensions analogues sont à l'œuvre dans l'exemple de La Chartreuse de Parme. L'apprêt « photogénique » des photographies de plateau, lourdement marquées par l'idéal de beauté extratemporelle recherchée par les

1 Victor Burgin, Indifferent Spaces. Place and Memory in Visual Culture. Berkeley, California University Press, 1995, pp. 22-23 (c'est nous qui traduisons). Le même auteur a prolongé ses recherches sur le thème des souvenirs cinématographiques dans son ouvrage The Remembered Film, Londres, Reaktion Books. On sait du reste que c'est aussi un thème cher à Stanley Cavell. Dans ce contexte, on peut mentionner aussi le livre de Peter Schwenger, Fantasm and Fiction : On Textual Envisioning, Stanford, Stanford University Press, 1999.

2 « Cinéma, histoire, novellisation: considérations autour d'écritures en miroir », in Alice Autelitano et Valentina Re (dir.), Il racconto del film/Narrating the film. La novellizzazione: del catalogo al trailer/Novelization: from the catalogue to the trailer (XII International Films Studies Conference), Universita degli Studi di Udine, 2006, p. 144

3 Id., pp. 144-145. 
studios Harcourt, n'est pas facilement conciliable avec l'effort stylistique de Pierre Véry fournissant un texte aussi «contemporain » et « neutre » que possible. L'auteur se vante en effet d'avoir fait parler les personnages selon les normes du jour et de s'être interdit tout caprice rhétorique :

Durant tout notre travail sur La Chartreuse, nous avons été guidés par la conviction que la plus forte marque de respect que nous pouvions témoigner à Stendhal était de le considérer comme un homme de notre temps, comme un des plus 'contemporains' de nos écrivains. Nous avons entretenu avec son œuvre des rapports de familiarité : nous avons conversé avec ses personnages comme avec des gens que l'on peut rencontrer dans la rue-dans nos rues de 1948- et nous avons fui comme peste l'imparfait du subjonctif. (...) Il va sans dire que, dans le présent texte, on s'est scrupuleusement interdit tout effort littéraire qui, après Stendhal, n'eût pu être qu'irrévérencieux, et parfaitement dérisoire'.

A la différence de la novellisation de Renoir, où le texte et l'illustration tirent l'œuvre cinématographique « vers le bas », ici l'inclusion des images « glamour » vise manifestement à tirer le texte de Véry « par le haut ", afin de le doter d'une caution culturelle et d'un minimum de patine historique dont le texte, violemment plat et anachronique, a grand besoin (après tout, c'est Stendhal qu'on adapte !) mais qu'il est incapable de produire à lui tout seul.

De telles analyses concernent et modifient autant le cinéma que la littérature. Aussi Arnoldy et Le Forestier ont-ils certainement raison de conclure :

Autour d'un de ses centres de gravité possibles, ici le cinéma, la novellisation a ainsi été abordée pour ce qu'elle participe aussi à faire l'histoire du cinéma. Loin de la restreindre à un remploi ou une récriture en seconde main d'un récit cinématographique, ces considérations nous ont progressivement conduit à aborder la novellisation en tant qu'elle est une 'mise en représentation' du film par l'écrit².

1 Pierre Véry, «Salutation à un vivant », in La Chartreuse de Parme, o.c., pp. 16-17.

2 « Cinéma, histoire, novellisation: considérations autour d'écritures en miroir », art. cité, p. 146. 
Jointe à la question de l'adaptation, cette seconde ligne de recherche conduit à complexifier encore davantage l'objet même des études cinématographiques. Les tensions entre le cinéma comme objet et le cinéma comme expérience vécue problématisent à leur tour le concept d' " original «. Elles demandent aussi que le cinéma soit vu à travers le prisme de ses prolongements visuels, souvent très éloignés de ce que l'on peut voir à l'écran.

La troisième et dernière interrogation qu'on voudrait présenter ici, forcément la plus brève, est en rapport avec les chemins que la novellisation n'a pas (encore) pris -ou qui semblent s'être transformées en autant d'impasses. Rien n'oblige en effet la novellisation à être d'abord textuelle. L'afflux d'images dans certaines novellisations illustrées indique qu'une adaptation photographique est pensable. Or, ce type de novellisation, dont La Jetée de Bruce Mau et Chris Marker représente un exemple isolé dans le champ de l'avant-garde, existe, ou du moins a existé, et même à grande échelle. Dans les années 50 du siècle dernier, on a connu en Italie -et dans une moindre mesure en France ${ }^{1}$ - un sousgenre croisant novellisation et roman-photo : les cineromanzi. ${ }^{2}$ Pendant quelque dix ans, l'industrie photoromanesque s'est emparée du cinéma pour diversifier une production alors au sommet de sa popularité. Le rendez-vous du roman-photo et de la novellisation était systématique, dans les deux sens du terme. D'une part, ce n'est pas un seul genre de cinéma, mais bel et bien le cinéma dans son ensemble qui se voyait adapté : des blockbusters hollywoodiens (Ben Hur de Wyler) aux comédies italiennes (Pain, Amour et...de Risi), des films de la Nouvelle Vague française (Les Cousins de Chabrol) aux œuvres post-existentialistes (Le désert rouge d'Antonioni), et ainsi de suite. D'autre part, tous ces films, tous ces genres, tous ces réalisateurs passaient sans exception au moule commun du roman-photo, avec sa mise en page stéréotypée et, surtout, avec son modèle narratif et idéologique tout-puissant, celui du mélodrame.

1 Pour un témoigage, voir Francesca Leonardi, "La Vie passionnée des Cousins: les avatars d'un film Nouvelle Vague", in Alice Autelitano et Valentina Re (dir.), Il racconto del film/Narrating the film. La novellizzazione: del catalogo al trailer/ Novelization: from the catalogue to the trailer (XII International Films Studies Conference), Universita degli Studi di Udine, 2006, pp. 179-195.

2 Voir Emiliano Morreale (dir.), Lo Schermo di Carta. Storia et Storie dei cineromanzi, Turin, Il Castoro, 2007. 
Ce corpus peu connu, que l'on vient seulement de redécouvrir grâce aux efforts de quelques collectionneurs privés, s'annonce comme une mine pour tout futur chercheur du cinéma, de la novellisation et des relations entre film et littérature. Deux grandes pistes se présentent tout de suite à l'attention.

Premièrement, la production des novellisations photoromanesques offre une belle occasion d'étudier dans le détail le jeu des réinterprétations visuelles du cinéma à une époque et dans un milieu déterminés, surtout si on arrive à mettre en rapport ces novellisations avec d'autres facettes, plus informelles et donc plus difficiles à reconstituer encore, de la culture visuelle ambiante. On peut penser ici aux photographies d'amateur, dont la circulation était intense (à en croire Giovanni Fiorentino, les tournages des films néoréalistes étaient ouverts au grand public comme aux professionnels locaux, qui avaient le droit de prendre, puis de vendre autant d'images qu'on voulait). ${ }^{1}$ Les cineromanzi italiens constituent donc un dossier exceptionnel pour quiconque aurait envie d'examiner sur pièces le phénomène de l'adaptation et de la mutation de l'objet-cinéma par son public même.

Deuxièmement, le même type de novellisation représente aussi un apport fondamental aux discussions plus larges sur la « littérature graphique ». Depuis quelques années, l'intérêt croissant pour la bande dessinée littéraire - dont le « roman graphique » n'est qu'une illustration parmi d'autres- a déplacé les enjeux du débat sur les relations entre littérature et bande dessinée. La grande question aujourd'hui n'est plus de savoir si telle ou telle forme de bande dessinée est en mesure ou non d'intégrer des aspects littéraires, mais si notre définition millénaire de la littérature comme art du langage tient toujours. Du moment qu'une bande dessinée muette, pour prendre un cas extrême, peut faire partie d'un corpus qualifié de littéraire, c'est l'être même de la littérature qui s'en trouve affecté. Toutes proportions gardées, les novellisations photoromanesques -et les novellisations illustrées en général-soulèvent des questions analogues. Elles brouillent les repères entre novellisation et roman-photo, entre littérature et cinéma, entre texte et image, exaspérant ainsi les mille et une hybridisations qu'explorent les théories de

1 Cf. Giovanni Fiorentino, Luci del Sud. Sorrento un set per Sofia, Naples, Eidos, 1995. 
l'adaptation, d'une part, et les études des rapports entre cinéma-objet et cinéma-expérience, d'autre part. 